\title{
Essais
}

ESSAIS

Revue interdisciplinaire d'Humanités

12 | 2017

Textes et contextes : entre autonomie et dépendance

\section{L'essence du divin et la dénomination : le personnage de Salvatore dans Il Nome della rosa, d'Umberto Eco}

Hynde Benachir

\section{(2) OpenEdition}

Journals

Édition électronique

URL : http://journals.openedition.org/essais/2858

DOI : 10.4000/essais.2858

ISSN : 2276-0970

Éditeur

École doctorale Montaigne Humanités

Édition imprimée

Date de publication : 15 juin 2017

Pagination : 101-127

ISBN : 979-10-97024-02-4

ISSN : $2417-4211$

Référence électronique

Hynde Benachir, «L'essence du divin et la dénomination : le personnage de Salvatore dans // Nome della rosa, d'Umberto Eco », Essais [En ligne], 12 | 2017, mis en ligne le 12 octobre 2020, consulté le 25 octobre 2020. URL : http://journals.openedition.org/essais/2858 ; DOI : https://doi.org/10.4000/ essais.2858 


\section{L'essence du divin et la dénomination : le personnage de Salvatore dans Il Nome della rosa, d'Umberto Eco}

\section{Hynde Benachir}

Umberto Eco affirme : «Rien n'est plus ouvert qu'un texte fermé1. ». Ce constat aux allures de paradoxe résume pourtant toute la tension créatrice qu'il peut exister entre le texte comme unité close et le texte comme unité fermée 2 . Récit, il est un signe clos, achevé dans sa temporalité discursive. Roman, il est un discours dont le sens demeure " en extension " ${ }^{3}$. Il est aussi l' " objet théorique ", lieu de cette " totalité seconde " que la sémiotique nomme " textualité ». C'est à partir de cette remarque, et sous l'angle de la conception de cette textualité comme une entité "irréductible ", que nous avons choisi d'aborder le roman, complexe s'il en est, d'Umberto Eco intitulé Il Nome della rosa.

Au-delà de la question du genre littéraire que sous-tend une œuvre d'une telle envergure, c'est la question même du texte, au sens étymologique du terme, qui transparait, notamment par la trame méta- et intertextuelle mise en place tout au long du roman. Citations glissées au détour d'un dialogue ou d'une description, locutions employées par l'instance narratrice ou par les personnages, rien de ce qui est " emprunté » à d'autres sources n'est traduit dans le texte d'Umberto Eco, quelle qu'en soit la langue source (latin, allemand, français, espagnol, grec, etc.). Véritable dédale linguistique, ce roman pose

1 Eco, Umberto, Lector in fabula, Paris, Grasset, 1979, p. 71

2 La notion de fermeture telle que nous l'entendons ici porte sur le rapport qu'il existe entre l'auteur et le lecteur. Si l'auteur est l'instance d'émission du discours, le lecteur est l'instance première d'interprétation de ce même discours. Le texte est "fermé " (la prévisibilité de la réception) dans la mesure où l'auteur pourrait lui donner une direction mais demeure " ouvert » sur l'extérieur, représenté par le lecteur et incluant les conditions de réception, la connaissance encyclopédique du lecteur, ses positions idéologiques, etc.

3 Nous reprenons ici la théorie du signe dans le langage poétique avancée par Julia Kristeva : "Terminer le roman en tant que récit est un problème rhétorique qui consiste à reprendre l'idéologème clos du signe qui l'a ouvert. Achever le roman en tant que fait littéraire (le comprendre en tant que discours = signe) est un problème de pratique sociale, de texte culturel, et consiste à confronter la parole (le produit, l'œuvre) avec sa mort - l'écriture (la productivité textuelle). ", in "Le texte clos ", Langages, $3^{\mathrm{e}}$ année, $\mathrm{n}^{\circ}$ 12, 1968, p. 124. 
ensuite la question de l'intelligible, laquelle se cristallise plus spécifiquement dans le personnage singulier de Salvatore, auquel nous nous intéresserons tout particulièrement pour ce travail. Moine bossu, ancien hérétique, sa difformité physique caractéristique semble trouver un écho dans le chaos linguistique dont s'alimente son langage. Rarement reformulées, sauf lorsque le contexte l'exige et moins à des fins de clarification que dans une progression actantielle précise, ses interventions dialoguées postulent un pacte tacite de compréhension entre Salvatore et ses différents allocutaires, fussent-ils intra-diégétiques ou non.

Or, ce langage se trouve être précisément le creuset des réponses aux interrogations que génèrent les différents niveaux de narration : c'est bien Salvatore qui connaît la raison première des meurtres perpétrés dans l'abbaye, tout comme il incarne le débat théologique qui doit avoir lieu entre les Franciscains et les légats du Pape au sujet de la pauvreté du Christ. L'ambivalence profonde de ce personnage l'élève donc au rang de symbole. Admirablement et fidèlement rendue dans l'adaptation filmique du roman par Jean-Jacques Annaud, la figure symbolique de Salvatore revêt à l'écran une importance toute particulière car le film, centré principalement sur l'intrigue policière, en fait un personnageclé dans le déroulement de l'enquête menée par Guillaume de Baskerville et son novice, Adso de Melk. C'est pourquoi nous retiendrons également cette adaptation filmique comme l'autre représentation dialogique de notre analyse, contexte multi-canal d'un même message où les domaines du para-verbal et de l'extra-linguistique peuvent effectivement être exploités.

La problématique de cette étude est donc double, à l'instar du binôme " texte/ contexte" : d'une part, il s'agit de comprendre comment cette langue inconnue, à la fois toute et aucune, devient universelle, et par là-même symbolique, dès lors que le personnage de Salvatore parvient à se faire comprendre de tous ses interlocuteurs, même si ceux-ci parlent différentes langues car venus de différents pays ; d'autre part, nous nous attacherons à montrer comment ces " lambeaux de langue " deviennent le puissant medium d'une polarisation axiologique où la créature bestiale et hérétique qu'est Salvatore au début acquiert peu à peu la grandeur du héros des mythologies païennes autant que du prophète des mythographies judéo-chrétiennes.

Enfin, si les deux premières problématiques énoncées semblent complémentaires, nous aborderons également celle, plus vaste, de l'auto-référence et de l'auto-réflexivité de l'œuvre du romancier italien que nous nous proposons d'analyser car, texte avant tout, elle est aussi un récit composé de plusieurs plans narratifs enchâssés les uns dans les autres, à l'image des langues qui se croisent et s'imbriquent dans le discours de Salvatore en posant la question suivante : si nous tenons que le texte ne saurait " parler " d'autre chose que du texte, de quoi nous " parle » Adso dans son récit? 


\section{Frontières de récits et récits de frontières}

Nous partirons d'un premier constat, qui touche au récit et à la situation d'énonciation tout à la fois. Le point de vue par lequel le lecteur aborde la diégèse est celui du narrateur-personnage, Adso de Melk, qui n'est cependant pas l'actant principal et qui se veut le plus neutre possible. Cette volonté d'objectivité est exprimée explicitement dès les premières lignes du récit, sous couvert d'une supplique quelque peu paradoxale : "Il Signore mi conceda la grazia di essere testimone transparente degli accadimenti che ebbero luogo all'abazia di cui è bene e pio si taccia anche ormai il nome, al finire dell'anno del Signore $1327[\ldots]^{4}$ ». La focalisation interne comme mode narratif semble entrer en contradiction avec l'objectivité du " témoin transparent » que postule le récit même si les termes employés par l'instance narratrice tendent à la distancier des événements relatés. En effet, les lexies "testimone ", "accadimenti ", tout comme la locution verbale "ebbero luogo" forment une isotopie du fait tel qu'appréhendé dans le cadre d'une enquête criminelle, laquelle constitue l'un des plans narratifs de l'œuvre d'Umberto Eco. Outre la controverse politicoreligieuse qui doit avoir lieu dans l'abbaye au moment où le narrateur et son maître, Guillaume de Baskerville, y séjournent, il incombe à ce dernier de résoudre le mystère des morts suspectes qui précèdent et suivent son arrivée entre les murs du monastère. La focalisation interne interviendrait alors comme un rapprochement tacite entre le narrateur-personnage et le lecteur, chacun ne sachant que ce que l'autre sait.

Cependant, une nuance intervient dans ce pacte naturel entre le narrateur en focalisation interne et le lecteur : l'utilisation d'un présent pour les verbes « $\grave{e}$ » et « si taccia» alors même que les autres verbes de la phrase qui se réferent au dit événement relaté relèvent des temps de l'inactuel, montrent un «décrochage » dans la chronologie du récit. Le temps de l'énonciation n'est pas celui de la narration. À l'instant où il "parle " - ici, nous savons que le narrateur écrit $^{5}$-, Adso sait déjà comment se termine son histoire. Au moment où il

4 Eco Umberto, Il Nome della rosa, Barcellona, Bibliotex, 2002, p. 13. Il existe une traduction française mais pour des raisons de clarté quant au propos de cet article, nous traduirons nous-mêmes les passages non traduits dans une volonté de rester au plus près du texte original. Traduction : Le Seigneur me concède la grâce d'être le témoin transparent des événements qui eurent lieu dans l'abbaye dont il est bon et pieux qu'on en taise encore aujourd'hui le nom, à la fin de l'an 1327(...).

5 La transcription du récit par le narrateur est clairement revendiquée dès le prologue du roman, dont nous avons extrait la première citation que nous avons reportée dans le texte. En effet, le personnage d'adresse directement au lecteur, qu'il rend complice de son secret, en l'appelant " mio buon lettore " en une sorte de captatio benevolentiae destinée à suspendre l'incrédulité de ce dernier et à mettre en place le pacte de lecture. Quant à la transcription, c'est toujours dans cette optique de complicité et d'identification entre lecteur et scripteur qu'elle est explicitée. Le narrateur, annonçant par prolepse le récit de faits terribles, adresse au lecteur plus qu’à lui même cette remarque sur l'acte d'écriture : "Ed è ora che, come noi allora facemmo, a essa si approsimi il mio racconto, e possa la mia mano non tremare nell'accingermi a dire quanto poi 
écrit, comme l'utilisation des temps de l'inactuel en témoigne, les faits qu'il transcrits appartiennent à un passé révolu que seul l'acte d'énonciation est en mesure de faire revivre. C'est pourquoi la présence de la locution adverbiale replace à la fois le discours dans la sphère du présent comme dans celle de la première personne, de même qu'elle a une incidence sur la relation entre le support et l'apport que représente le « je " de la voix narratrice et le verbe "si taccia ». Sous couvert d'un acte " pieux " et " bon ", le narrateur-scripteur dévoile une manipulation de son discours visant à cacher des renseignements comme ici le nom de l'abbaye. Ainsi, le lecteur est prévenu : s'il peut avoir une connivence réelle avec le Adso du récit, il ne peut en revanche se faire fort de savoir ce que le Adso de l'énonciation sait.

Cette remarque nous amène donc à considérer, comme second constat, le récit d'Adso et son cotexte, puis, plus globalement, le récit dans son contexte. À ce titre, il est important de noter que le roman ne correspond pas au seul récit du narrateur-personnage mais se présente plutôt comme une stratification de récits, introduits par divers actants de l'activité narrative.

En effet, le récit à proprement parler est introduit par un prologue de la main du narrateur-personnage dont nous avons extrait la citation que nous venons de commenter. Ce prologue, en dépit des prolepses narratives dont il est émaillé, n'est qu'une grande analepse où les faits sont contextualisés par rapport à la chronologie diégétique : le lecteur est explicitement averti de ce que les faits rapportés ne sont pas concomitants au temps de l'énonciation. Le livre que le lecteur tient dans les mains devient alors l'objet même de ce qu'il lit, la matérialisation de l'acte d'écriture revendiqué dès les premières lignes du prologue et sur lequel ce même prologue s'achève. Ensuite, si nous étendons le récit au texte rythmé par les heures et à son prologue, il nous faut dès lors prendre en considération un autre texte, rendu en italique dans le livre et intitulé "Naturalmente, un manoscritto ", de la main de l'auteur lui-même. Le mot "manoscritto ", par dérivation impropre, révèle de façon éminemment auto-référentielle la nature même du texte que le lecteur sera amené à prendre en compte, si bien qu'à cet endroit de la narration, ce dernier se trouve déjà face à un dilemme portant sur le manuscrit : s'agit-il de celui d'Adso ou de celui de l'auteur lui-même ? Si nous partons de l'hypothèse que cette note datée du 5 janvier 1980 est bien une incursion illocutoire de l'auteur, le récit d'Adso est donc au second niveau d'un enchâssement narratif dans lequel Umberto Eco raconte ce qu'Adso raconte. Or donc, cette note est plus qu'une simple volonté de l'auteur de se manifester puisqu'elle annonce

accadde. ", in Eco Umberto, op. cit., p. 19. Traduction : Et maintenant que, comme nous fimes alors, l'heure de mon récit approchant, puisse ma main ne pas trembler tandis que je me prépare à dire ce qui se passa alors...

6 Traduction : Naturellement, un manuscrit. 
deux autres niveaux de narration en citant les différents ouvrages ayant trait au récit d'Adso puisque les écrits autobiographiques du moine autrichien ayant vécu au XIV ${ }^{\mathrm{e}}$ siècle ont d'abord été compilés et traduits par deux personnages différents : "Il 16 agosto 1968 mi fu messo tra le mani un libro dovuto alla penna di tale abate Vallet, Le Manuscript de Dom Adson de Melk, traduit en français d'après l'édition de Dom J. Mabillon (Aux Presses de l'Abbaye de la Source, Paris, 1842) ${ }^{7}$.". Plusieurs points doivent ici attirer notre attention. Tout d'abord, force est de constater que l'ancrage temporel revêt une importance particulière car chaque étape de l'entrée dans le récit est datée assez précisément de sorte que le lecteur, ouvrant le livre même bien des années après sa publication, sera toujours confronté à un temps fini mais non clos, comme si le témoignage qui se trouvait sous ses yeux n'avait de finitude que dans la sphère textuelle pure et non dans la réalité de sa lecture. Cette chronologie qui lui échappe l'entraîne pourtant de loin en loin dans un temps historique, et le terme est ici à prendre dans son sens le plus large.

De fait, ces considérations semblent importer bien moins que les sources qui sont mentionnées et ce n'est sans doute pas un hasard si la traduction de l'Abbé Vallet a été publiée aux Presses de l'Abbaye de le Source. Enfin, si le récit est bien de l'Abbé Vallet, celui-ci n'est que troisième de liste puisqu'il ne fait que traduire un texte compilé par Dom J. Mabillon et écrit par Adso de Melk. S’inscrivant dans cette dynamique, le lecteur est alors cinquième de liste à prendre connaissance des écrits du moine autrichien. Une fois encore, la posture du lecteur s'avère ici ambiguë : doit-il attribuer le manuscrit qu'il lit à Adso s'il a, au préalable, pris connaissance de la note mentionnant le rôle joué par Dom J. Mabillon, par l'Abbé Vallet, par l'auteur lui-même ? Loin de rester dans son rôle de destinataire extra-diégétique, le lecteur se voit contraint d'échafauder lui-même les hypothèses nécessaires à la compréhension la plus exhaustive possible du récit devant lequel il est placé et entre lui aussi dans la spirale des lecteurs des écrits premiers. Ainsi, à l'idée de citation qui s'impose avec l'allusion aux diverses étapes traversées par les écrits d'Adso, se surimpose logiquement la notion concrète d'intertextualité :

J'ai redécouvert ainsi ce que les écrivains ont toujours su (et que tant de fois ils nous ont dit) : les livres parlent toujours d'autres livres, et chaque histoire raconte une histoire déjà racontée. Homère le savait, l'Aristote le savait, sans parler de Rabelais ou de Cervantès. C'est pourquoi mon histoire ne pouvait que commencer par le manuscrit retrouvé, c'est pourquoi cette histoire aussi

7 Eco Umberto, op. cit., p. 5. La citation a été transcrite dans le présent travail telle qu’on la trouvera dans le texte, en respectant la casse. «Le 16 août 1968 il me fut mis entre les mains un livre dû à la plume d'un certain abbé Vallet (...)». Nous aurons à cœur de noter la tournure impersonnelle employée par " l'auteur » et sa passivité, voire son inactivité, devant la restitution des écrits d'Adso de Melk. Le livre lui a été « mis entre les mains » et il n'en est pas l'auteur primordial sans que l'on sût qui le lui avait remis et ni dans quelles circonstances. 
serait une citation (naturellement). J'écrivis tout de suite l'introduction, plaçant ma narration à un quatrième niveau d'emboîtement, à l'intérieur des trois autres niveaux de narration : moi je dis que Vallet disait que Mabillon a dit que Adso $\operatorname{dit}^{8} \ldots$

Contraint sans sa finitude comme récit, le texte regagne sa plus grande extension dès lors qu'il est considéré comme une citation. Synecdoque d'un texte autre, la citation est à la fois signifiante par-elle même et porteuse du sens du contexte-source dont elle est tirée, mais acquiert un sens autre au contact du contexte-cible dans lequel elle est replacée. Vecteur d'un sens en acte, elle se définit comme cristallisation d'un double mécanisme linguistique intrinsèque à la production langagière : celui de la répétition et celui de la métaphore. Si la première activité de l'Homme en matière de langage est de répéter ce qu'il entend, la seconde est de réinvestir ce qu'il aura répété. De fait, l'auteur doit, dans l'Apostille publiée cinq ans après la première publication du roman, revenir sur le mécanisme fondamental de son écriture de même qu'il doit en expliciter le but ${ }^{9}$.

Cette poétique de la citation se fait jour dès les premières lignes du récit d'Adso, reprenant le premier verset de la Genèse ${ }^{10}$, et se poursuit jusqu'à la dernière ligne de sa transcription puisque le texte s'achève sur la citation d'un hexamètre en latin extrait de l'ouvrage de Bernard de Morlaix, De Contemptu mundi dont la thématique principale est l'ubi sunt : " [...]stat rosa pristina nomine, nomina nuda tenemus ${ }^{11}$. ". Cette rose dont il est fait mention par le bais de la citation du moine bénédictin français qui vécut au XII ${ }^{e}$ siècle n'a d'autre écho que la rose mentionnée dans le titre car, à aucun moment dans le récit, il n'est fait état - à première vue - d'une rose ou quelque autre objet que ce fût qui puisse la métaphoriser ou la re-présenter ${ }^{12}$. Le titre du

8 Eco Umberto, Apostille au "Nom de la rose ", Paris, Grasset, 1985, p. 25.

9 Cette stratification du récit est perceptible à travers la gradation temporelle mise en ouvre lorsqu'il rappelle le rôle joué par les différentes instances, lectrices puis narratrices, allant du présent, en passant par l'imparfait puis le passé composé, avant de revenir au présent non plus de l'énonciation mais du récit.

10 Umberto Eco, op. cit., p. 5. Le verset de la Bible auquel nous faisons référence apparait dans le Prologue comme suit : "In principio era il Verbo e il Verbo era presso Dio, e il Verbo era Dio.". Traduction : Au commencement était le Verbe, et le Verbe était auprès de Dieu, et le Verbe était Dieu. Rien dans le texte n'identifie explicitement la source de cette citation. Aussi, nous en déduirons que, comme pour toute citation, le narrateur fait ici appel à un référentiel commun partagé avec le lecteur, lequel doit recourir à l'exophore mémorielle afin de mieux comprendre à quel texte "absent » il est fait allusion dans le texte qu'il lit.

11 Eco Umberto, ibid., p. 473. Traduction : La rose originelle n'existe que par son nom, et nous n'avons que des noms dépouillés [de sens]. Devant la syntaxe hautement elliptique de cette citation, nous proposons cette fois encore une traduction au plus près du sens. Quant au topique de l'ubi sunt mentionné ici dans une visée linguistique, il rappelle l'idée que le langage peut désigner des choses existant encore comme des choses passées ou abolies. Ainsi, il est dans le langage des objets "passés " dont nous ne possédons plus que le nom.

12 Cette remarque doit toutefois être nuancée. La jeune fille qui fait une brève apparition dans le 
roman n'est pas pour autant "déconnecté » de son essence puisqu'il postule un relation bien spécifique du texte à son signifiant : le sens n'est en somme que la résultante plus ou moins volontaire d'un assemblage de signifiants dont l' "immanence » rend le texte perceptible comme une entité autonome et " irréductible ${ }^{13}$ ». Moins qu'un nom, ce titre met en exergue la relation au nom que se devra d'entretenir le lecteur avec le texte s'il veut entrevoir le sens véritable de ce qui lui est donné à lire.

À la circularité du récit ouvert et fermé par la citation, à sa nature explicitement revendiquée de citation des écrits autobiographiques d'Adso de Melk, s'ajoutent également toutes les citations qui émaillent le texte et dont la source n'apparaît pas toujours clairement. Au détour d'un dialogue, d'une pause narrative, les textes semblent surgir de la mémoire du narrateur et se glisser dans la trame de son récit sans qu’on y prît garde ou presque. Cependant, ces " intrusions " se laissent appréhender sans difficulté puisque, dans tous les cas, les citations ne sont jamais traduites quand elles sont actualisées et sont mises en italique dans le texte. La langue du texte devient alors elle-même un faisceau de signifiance, agissant comme une balise visant à montrer à l'allocutaire, le "mio buon lettore ${ }^{14}$ " à qui s'adresse directement le narrateur-personnage, que la voix d'Adso est momentanément suspendue au profit d'une autre - voix d'autorité extérieure - et qu'il lui revient de l'identifier comme telle avant de lui restituer son origine s'il le peut.

Nous insistons sur cet "échec " de l'allusion auquel sera nécessairement confronté le lecteur car il est ménagé dès le départ par la voix narrative. Comme nous l'avons vu, le narrateur-personnage n'est pas à pied d'égalité avec le lecteur, bien qu'il cherche à lui faire accroire le contraire, notamment au moyen de cette "fausse " focalisation interne que nous avons identifiée. L'information, et avec elle le sens, sont distillés par la seule instance narratrice, la même qui cite et fait allusion. Se pose alors la question de la véracité relative des allusions dont elle jalonne le texte : si le pacte inclut que le lecteur devra croire ce qui lui est raconté, il devra également croire la manière dont les faits lui sont racontés. En somme, ce qui s’applique au contenu du texte s'applique

récit - et dont Adso tombe amoureux - fait partie du schéma actantiel mais à aucun moment le lecteur ne voit apparaitre son nom. Dans l'adaptation cinématographique, la voix off rappelle ce détail avant que n'apparaisse la citation en latin à l'écran. Il se peut donc que l'un des objets représentés symboliquement par la rose soit cette jeune fille existant, mais non nommée. En outre, la rose peut également être interprétée comme un renvoi intertextuel au Roman de la rose (aussi bien à la version de Jean de Meung qu’à celle de Guillaume de Lorris). Enfin, sans doute la rose des Sonnets pour Hélène (parus en 1578), de Jean de Ronsard, trouve également un écho dans ce récit où tout ne semble qu'illusion, vanité et mortalité.

13 Nous reprenons, pour plus de précision dans notre analyse, la terminologie employée par Frédéric Bravo lors de son intervention dans le cadre de la Journée d'études sur le binôme « texte/contexte " qui a eu lieu en mai 2014 à l'Université Bordeaux Montaigne.

14 Traduction : mon bon lecteur. 
également à sa substance. Quid de cette substance dont on sait d'emblée qu'elle n'est que le miroitement d'un texte autre, amputée par endroits de certains renseignements? Tout comme le narrateur tait le nom de l'abbaye, l'auteur tait les circonstances dans lesquelles il a eu connaissance des écrits du narrateur, tout comme nous ne savons pas comment Dom J. Mabillon s'est procuré à l'époque le manuscrit d'Adso, tout comme les écrits d'Adso reposent fondamentalement sur la quête d'un livre qui n'existe pas. En effet, c'est parce que nous savons que le second tome de la Poétique d'Aristote (et le philosophe grec revient sous la plume du romancier italien lorsqu'il parle d'intertextualité) n'existe pas que nous devinons que le manuscrit retrouvé, puis perdu de nouveau, n'existe pas non plus. Lorsque l'auteur raconte qu'il avait emporté le manuscrit d'Adso dans ses valises lors d'un séjour à Prague et que celui-ci lui fut " dérobé » par inadvertance par l'" être cher » qu'il devait retrouver dans la capitale tchèque, il ne fait que déléguer l'autorité au narrateur qu'il devient en posant les fondations de sa fiction. Nicolas Rieder, dans l'article qu'il consacre à l'intertextualité dans le roman d'Umberto Eco, définit cet univers textuel comme une "bibliothèque fantôme ${ }^{15}$ ". Dans son ouvrage intitulé La Fiction, Christine Montalbetti revient elle aussi sur ce type de préface dont le point de départ est une trouvaille de manuscrits :

Lieu où tremble le statut du texte, et pourtant lieu fortement codé, qui dit "Ceci est référentiel " mais que, tel le pacte réaliste, je ne dois sans doute pas prendre à la lettre, que je dois reconnaître comme un surcroît de fiction, qui travaille à constituer une fiction seconde et introductive, qui porte sur le régime du texte que je m’apprête à lire ${ }^{16}$.

En annonçant judicieusement le vacillement du "statut du texte ", Christine Montalbetti nous pousse à considérer plus profondément le statut du cotexte. Ces " préfaces fictionnelles ", outre la " mise en fiction " dont elles sont investies, agissent alors comme un manifeste poétique, havre de conscience herméneutique qui donne au lecteur les clés nécessaires à l'entendement du discours tel que donné et du discours tel qu'entendu. Tous les textes mentionnés dans le récit n'existent pas forcément, à commencer par celui sur lequel le récit lui-même se fonde. La notion de cotexte devient alors artéfactuelle et se laisse voir comme telle. Ce méta-récit, et le terme apparaîtrait presque ici comme une tautologie tant son auto-référence est prégnante, prend toute sa dimension dès lors qu'on apprend, en lisant ce Prologue, que les écrits d'Adso de Melk ne sont restés que six jours en possession de l'auteur avant d'être à nouveau perdus, temps durant lequel ce dernier aura pris des notes. Ce qui n'est pas sans rappeler que le compte des jours qui égraine le récit

15 Rieder Nicolas, «Finis Africae : dans la bibliothèque fantôme d'Umberto Eco », Acta Fabula, automne 2014.

16 Montalbetti Christine, La Fiction, Paris, Flammarion, 2001, p. 162. 
d'Adso dans l'abbaye "fantôme " s'arrête lui aussi à six tandis que les morts suspectes sont également au nombre de six. D'un point de vue symbolique, le temps de la lecture des écrits autobiographiques par l'auteur correspond au temps de la diégèse donné par le narrateur. De là à dire que l'un et l'autre ne font qu'un, il n'y a plus qu'un pas vite franchi dès lors que l'on se souvient que le roman s'ouvre sur une citation de la Genèse, récit de la Création du Monde qui dura six jours. Plus que lecteurs, nous devenons alors spectateurs de la création d'un monde sous nos yeux, au fil des lignes qui tendent vers une fin tragique annoncée dès le début ${ }^{17}$.

\section{Salvatore thaumaturge}

Nous tenons donc que si le langage est affaire de signifiants, les langues seraient alors affaire de signifiance. Cette mosaïque de textes devient alors kaléidoscope de langues où, dans un même syntagme, différents systèmes linguistiques s'imbriquent les uns dans les autres avec, pour toile de fond, la langue du contexte qu'est l'italien. Les citations en latin, en français ou en allemand voisinent immédiatement avec le texte où elles se trouvent insérées, en une sorte de " patchwork " idiomatique qui pousse le lecteur à prendre chaque pièce isolément pour les considérer ensuite comme un tout. Même la graphie revêt une importance capitale dans ce camaïeu narratif puisque les citations extraites du second tome de la Poétique sont transcrites par le traducteur de grec, Vénantius de Salvemec, en alphabet zodiacal sur un parchemin qui, comme tous les manuscrits de l'histoire, sera retrouvé par Guillaume de Baskerville lors de son enquête dans l'abbaye. Le paradoxe touche presque à son comble puisque le " texte fantôme " d'Aristote se trouve encodé dans un alphabet aussi hermétique que l'ancien inquisiteur devra à son tour déchiffrer tant pour Adso que pour le lecteur incapable de le lire en dépit du fait que la voix narratrice ait pris la peine de le transcrire fidèlement. Pour cet exemple encore, il ne s'agit que de mettre en relation, dans l'axe des successivités, divers systèmes de signes à ceci près que l'alphabet zodiacal n'est que symboles et ne transcrit aucune langue : il est juste un autre code avec lequel le lecteur doit composer.

17 Si l'allusion à la Genèse apparaît de manière claire dans l'incipit du roman, une allusion à l'Apocalypse de Saint Jean est alors sous-entendue dans le même incipit. En effet, les faits tragiques évoqués dans l'analepse fait par Adso dès les premières lignes laisse entendre une fin chaotique, effective avec l'incendie qui détruit la bibliothèque, la mort de deux autres moines brûlés sur le bûcher, la rébellion qui s'empare des paysans vivant sous la domination de l'abbaye. Il est par ailleurs bon de noter que, lors des premiers meurtres, Ubertin de Casale cite les versets de l'Apocalypse pour prédire les circonstances et les conséquences des morts qui suivront. 
L'apogée de cette imbrication, qui relève alors moins du " patchwork " que d'un vrai tissage sémiotique, $s^{\prime}$ incarne dans le personnage de Salvatore ${ }^{18}$. Â l'instar de l'abbaye dans laquelle il se trouve, ce personnage n'est désigné que par ce prénom, qui pourrait ne pas être le sien étant donné qu'il a fui son village d'origine ravagé par des épidémies alors qu'il était enfant. De façon générale, les moines sont, selon la coutume de l'époque, désignés par leurs prénoms auxquels on accole le lieu de leur provenance mais ce n'est pas le cas du moine difforme : s'il ne vient de nulle part, en vertu d'un postulat linguistique bien admis selon lequel tout locuteur doit être ancré dans l'espace pour assumer un discours, Salvatore pourrait être lui aussi un spectre sans repères, dont le discours n'est plus soumis à aucune loi. Par ailleurs, si le lecteur prend conscience que cette exception ne fait que confirmer la règle, il pourra également s'interroger sur le nom de Guillaume de Baskerville. Il est tout à la fois une référence à Guillaume d'Ockham, dont les théories sur le nominalisme et le conceptionnalisme sont largement représentées alors même que le personnage du roman d'Umberto Eco avoue éprouver amitié et admiration pour le philosophe, et au roman Le Chien des Baskerville d'Arthur Conan Doyle, dont le détective Sherlock Holmes a posé les jalons de la logique inductive dans le roman policier, logique dont se sert l'ancien inquisiteur pour faire la preuve des hypothèses qu'il avance ${ }^{19}$.

Toujours dans cette optique onomastique, le fait que l'identité du personnage de Salvatore ne soit réduite qu'à son simple prénom permet de canaliser l'attention du lecteur, qui n'associe rien d'autre au personnage que la substance qui l'alimente. Ainsi, allié du mystère qui entoure l'existence de cet être particulier, ce prénom seul peut laisser apparaître son sens et peut également laisser entrevoir, de façon métonymique, la figure christique du «Sauveur » en un lieu décrit dès les premières lignes comme plongé dans le chaos. Tributaire de l'image qu'il renvoie comme de celles qui l'entourent, Salvatore apparaît pour la première fois dans le récit alors que le narrateur-personnage est en train d'admirer les sculptures effroyables du chapiteau d'un des portails de l'église de l'abbaye, où figurent toutes sortes de créatures imaginaires : "L'essere alle nostre spalle pareva un monaco, anche se la tonaca sudicia e lacera lo faceva

18 Nous noterons l'importance de ce simple prénom pour sa définition onomastique : caractérisé comme le "sauveur ", il apparaît que le rôle presque messianique joué par le moine bossu est postulé d'emblée par le nom qu'il porte - ou dont on l'a baptisé.

19 La théorie de la logique inductive rappelle immanquablement un autre principe ockhamien : celui de l'économie cognitive, également connu sous le nom de " rasoir d'Ockham " ou principe d'économie cognitive. Le philosophe établit qu'il n'est pas besoin de multiplier ce qui est pour prouver l'existence lorsque ce n'est pas nécessaire. Ainsi, c'est à partir de ce que nous avons que nous devons raisonner, et non multiplier les faisceaux du raisonnement. Ce principe est illustré par l'anecdote du cheval perdu de l'Abbé alors que Guillaume arrive tout juste à l'abbaye : avec la seule empreinte du sabot découverte dans la neige, Guillaume donne une description précise du cheval, devine son nom et, bien entendu, l'endroit où il se trouve. 
assomigliare piuttosto a un vagabondo, e il suo volto non era dissimile da quello dei mostri che avevo appena visto sui capitelli ${ }^{20}$.". Selon la voix narratrice, ce n'est pas seulement la circonstance qui rapproche le personnage de Salvatore des chimères sculptées dans la pierre de l'édifice religieux : c'est en effet son caractère indubitablement fantastique, parangon de laideur tel qu'il semble à peine croyable, qui l'élève au rang de créature onirique.

Cette incrédulité n'est pas sans rappeler celle que l'on demande au lecteur de mettre de côté dans le pacte tacite de la lecture. En utilisant la première personne du pluriel à ce moment précis du récit pour localiser le personnage de Salvatore dans le syntagme nominal " alle nostre spalle », le narrateur semble enrôler le lecteur dans le récit et, le mettant " dos à " la réalité, l’oblige en quelque sorte à admettre celle-ci. Ce " nous " est moins une tournure visant à atténuer l'autorité de la voix narrative qu'une manière explicite de "gommer " toute éventuelle frontière entre le contexte du récit et celui du lecteur, l'un demeurant dans la diégèse alors que l'autre doit irrémédiablement rester au-dehors. Le champ lexical de l'apparence est largement représenté dans cette introduction du personnage de Salvatore dans le récit : "pareva", " assomigliare ", " dissimile ". Il est, par ailleurs, intéressant de constater que "volto » trouve dans le texte deux synonymes : "fatezze» (comme hyponyme) et "faccia» (comme co-hypéronyme $)^{21}$.

20 Eco Umberto, op. cit., p. 46. Traduction : L'être derrière nous paraissait [être] un moine, même si la tunique sale et déchirée le faisait plutôt ressembler à un vagabond, et son visage n'était pas différent de ceux des monstres que je venais à peine de voir sur les chapiteaux. Ici, le mot "dissimile " revêt une importance particulière puisqu'il est le dernier des mots du champ lexical à apparaître dans le syntagme phrastique. Si son sens se voit neutralisé par la négation du verbe d'état qui le précède, ce n'est pourtant pas " differente » qui est actualisé dans le texte. La motivation de ce choix semble être le potentiel évocateur de chacun. Le lexème "differente » est un étymon latin (dif-fero) tandis que "dissimile " est un lexème formé dans la langue italienne (préfixe privatif « dis- " accolé au radical «simile »). Si les deux mots sont porteurs du même préfixe " di-» infirmant ce que le radical dénote, "-fferente " lu seul est nettement moins évocateur que le mot "simile » qui apparaît dans son entier une fois le préfixe écarté. D’un registre plus soutenu, le lexème "dissimile " devient plus pertinent encore puisque l'étymon italien reste directement accessible à tout locuteur italophone. De fait, presque comme un énantiosème, le mot «dissimile " se pose comme une jonction où se rencontrent un sens et son contraire, l'un inféodé au radical et l'autre à la modalisation pour ne retenir au final que la similitude effective entre le moine ("pareva un monaco» au début de la phrase) et les monstres ("non era dissimile dei mostri » à la fin de cette même phrase) qui trouvent tous deux dans leurs cotextes immédiats un élément du champ lexical de l'apparence.

21 Eco Umberto, ibid., p. 47. L'analogie entre les monstres du portail et le personnage de Salvatore revient sous la plume d'Adso, après que ce dernier a reporté les premières paroles du moine avec quelques nuances toutefois : "In quel momento, in cui lo incontrai per la primera volta, Salvatore mi apparve, e per il volto, e per il modo di parlare, un essere non dissimile dagli incroci pelosi e ungulati che avevo àppena visto sotto il portale. ". Traduction: À cet instant, où je le rencontrai pour la première fois, Salvatore m'apparut, et par son visage, et par sa manière de parler, comme un être non dissemblable des hybrides poilus et griffus que je venais juste de voir sous le portail. Outre la paronomase entre "volta " (fois) et "volto " (visage) qui relie de façon syntag- 
Dans l'adaptation filmique du roman par Jean-Jacques Annaud ${ }^{22}$, cette représentation de la première apparition de Salvatore se fait en vertu de l'esthétique médiévale : au premier plan, l'ombre insolite du moine se détache, plus grande et plus grosse, sur le fond des sculptures du chapiteau grâce à la profondeur de champ (0:27:25). Salvatore, caché derrière une colonne, est plongé dans l'obscurité tandis qu'avant même de prononcer la moindre parole, il se met à rire. Dans cette abbaye où le rire est un crime, le spectateur voit alors se profiler un contre-pouvoir exercé par ce personnage qu'il aura bien vite caractérisé comme dissident et différent. La comparaison avec le Diable évoquée dans le texte d'Umberto Eco sous la plume d'Adso ${ }^{23}$ est relayée à l'image où Salvatore montre le Diable sur les sculptures avant d'imiter la tête cornue apparue à l'écran. De même, si le rire de Salvatore se fait entendre dans le film, son sourire est présent dans le texte, juste avant qu'il ne commence à parler ${ }^{24}$.

Ces « ricochets » lexématiques, qui confèrent au texte un aspect cyclique, permettent également une fusion de la parole d'Adso, qui habite le texte, et de celle de Salvatore, qui s'y invite. Ainsi, lorsque le narrateur décrit " notre " interlocuteur, il parle de sa tête rasée et, dans la description ${ }^{25}$, se glisse le mot

matique la chimère au domaine de l'empirique, le texte prend ici une allure performative. Effectivement, rien n'est dissemblable du texte précédent dans ce qui parle de la ressemblance alors que tout le reste est modifié. On y trouve le même mot "essere " qui à la fois annonce le statut et l'apparition réelle de cette créature, de même que l'adverbe "appena " (juste) dont l'incidence rapproche le passé de l'énonciation si près du locuteur - et de son allocutaire - qu'il redouble encore l'effet de cette répétition aux airs d'antanaclase.

22 Le Nom de la Rose et L'abbaye du crime, réalisé par Jean-Jacques Annaud, Neue Constantin Film Production GmbH et Warner Bros. Enterntainment Inc., 2004.

23 Eco Umberto, op. cit., p. 46 : "Non mi è mai accaduto, come invece accade a molti miei confratelli, di essere visitato dal diavolo, ma credo che se esse dovesse apparirmi un giorno, incapace per decreto divino di celare appieno la sua natura anche quando volesse farsi simile all'uomo, esso non avrebbe altre fattezze di quelle che mi presentava in quell'instante il nostro interlocutore.". Traduction : Il ne n'est jamais arrivé, comme il arriva à nombre de mes confrères, d'être visité par le diable, mais je crois que sill devait m'apparaître un jour, incapable par décret divin de cacher totalement sa nature quand même il voudrait se faire pareil à l'homme, celui-ci n'aurait d'autres traits que ceux que me présentait à cet instant notre interlocuteur. Nous attirons l'attention sur deux points importants. Le premier est l'apparition du verbe "celare " alors même que le personnage apparaît caché dans le film. Le second porte sur "il nostro interlocutore ", qui place explicitement le lecteur dans la même situation d'interlocution que le narrateur.

24 Eco Umberto, ibid., p. 46. Adso écrit : "L'uomo sorrise (o almeno cosi credetti) [...]". Traduction : L'homme sourit (ou c'est ce que je crus) [...]. Aussitôt suivi d'une parenthèse illocutoire de la voix narratrice, le verbe "sorrise " apparait clairement dans le texte et son sujet n'est plus "l'essere " (l'être), par trop général et peu caractéristique : il semblerait, à ce stade de la description du personnage, que le rire fasse de lui un homme et que ce rire devienne le propre de l'Homme, comme l'aura dit Aristote. C'est précisément parce qu'il rit que la figure monstrueuse de Salvatore acquiert sa dimension humaine.

25 Eco Umberto, ibid., p. 46 : "La testa rasata, ma non per penitenza, bensi per l'azione remota di qualche viscido eczema [...]" Traduction : La tête rasée, non par pénitence, mais plutôt par l'effet ancien d'un eczéma sournois [...]. 
"penitenza" (pénitence) qui revient dans la bouche de Salvatore alors qu'il prononce le premier mot de son monologue : "Penitenziagite! " ".

Afin de mieux percevoir le " patchwork " idiomatique que nous évoquions plus haut, nous nous proposons de reporter ci-dessous la totalité de cette première intervention de Salvatore :

Penitenziagite! Vide quando draco venturus est a rodergarla l'anima tua! La mortz est super nos! Prega che vene lo papa santo a liberar nos a malo de todas le peccata! Ah ah, ve piase ista negromanzia de Domini Nostri Iesu Christi! Et anco jois mes dols e plazer m'es dolors... Cave el diabolo! Semper m'aguaita in qualche canto per adentarme le carcagna. Ma Salvatore non est insipiens! Bonum monasterium, et aqui se magna et se priega dominum nostrum. Et el resto valet un figo seco. Et amen. No ${ }^{27}$ ?

Plusieurs constats sont à faire dans cette première intervention directe du personnage. Le premier concerne l'énonciation : tel l'enfant dans les premières phases du langage, le moine ne se sert pas de la première personne du singulier pour parler de lui-même mais dit son prénom et passe, de fait, par une troisième personne du singulier. Ce recours à la personne délocutée du dialogue ne va pas sans une certaine mise à distance de la parole, comme s'il se refusait ou ne pouvait en assumer l'acte. Cette hypothèse se trouve partiellement confirmée dans le commentaire que fait Adso au sujet de ce qu'il appelle "favella" et non "lingua ", plaçant le système linguistique employé par Salvatore à un stade infra-idiomatique, soit un système cohérent dans sa logique langagière mais incohérent du point de vue de la langue :

Mi avvidi pure in seguito che egli poteva nominare una cosa ora in latino ora in provenzale, e mi resi conto che, più che inventare le proprie frasi, egli usava disiecta membra di altre frasi, udite un giorno, a seconda della situazione e delle cose che voleva dire $e^{28}[\ldots]$

Si la syntaxe du monologue de Salvatore se trouve résolument placée sous l'égide de la parataxe, c'est sans doute parce qu'il ne peut faire autrement. Tout ce qu'il exprime, comme le souligne le narrateur, n'est en fait que citations de conversations entendues et dont les éléments sont utilisés ad hoc.

26 Eco Umberto, ibid., p. 46. Ce mot fera l'objet d'une explication dans une note ultérieure.

27 Eco Umberto, ibid., p. 46. Devant les nombreuses langues employées ici disjecta membra, nous proposons la traduction suivante en français : "Penitenziagite! Vois comme le serpent est prompt à ronger ton âme! La Mort est sur nous! Prie pour que vienne le pape saint nous libérer du mal de tous les péchés! Ah ah, elle vous plait, cette nécromancie de Notre Seigneur Jésus-Christ ! Et de même que la joie m’est deuil le plaisir m’est douleur ! Attention au Diable ! Toujours il me guette dans un coin pour planter ses dents dans mes talons. Mais Salvatore n'est pas ignorant! [C'est] un bon moine, et ici on mange et on prie notre seigneur. Et le reste vaut une figue sèche. Et amen. Non?".

28 Eco Umberto, ibid., p. 47. Traduction: Je m’aperçus par la suite quil pouvait nommer une chose tantôt en latin tantôt en provençal, et je me rendis compte que, plus qu'inventer ses propres phrases, il utilisait des morceaux épars d'autres phrases, entendues un jour, selon la situation et ce qu'il voulait dire $[. .$.$] .$ 
L'inventio platonicienne est celle du moine tandis de la dispositio ne lui appartient plus sans que l'on sût jamais de qui elle émanait, Salvatore devenant source première d'un texte qui n'est pourtant pas le sien. Ainsi, son rôle dans le discours se borne à l'actualisation de quelques connecteurs logiques qui, à leur tour, entrent en contradiction avec l'incohérence logique du contenu discursif. Le "et " n'apparaît qu'au milieu de son monologue, à deux reprises, et revient à la fin en guise de conclusion, faisant de cette conjonction de coordination un artifice "sommaire » dans le fil de son discours. Le " $m a^{29}$ " adversatif précède son propre prénom, semblant montrer que le seul point sur lequel il peut raisonner de façon construite n'est autre que lui-même. Par ailleurs, le narrateur emploie le verbe "nominare ${ }^{30}$ " pour désigner l'action non pas de nommer mais d'actualiser dans une langue ou dans une autre tel mot que Salvatore voudra prononcer, lui reconnaissant par là-même une fonction thaumaturgique somme toute particulière : le vecteur de sens n'est plus le mot mais bien la langue dans laquelle le mot est exprimé, choix relevant exclusivement du locuteur et enfreignant les règles qui définissent le fonctionnement d'une langue comme système clos et autonome. Le langage de Salvatore, s'il se laisse comprendre - et c'est là la condition majeure de sa définition -, ne peut être pris comme une langue bien qu'il soit cependant un système.

Ainsi, nous pouvons voir se dessiner dans le discours du moine bossu plusieurs sphères linguistiques. La sphère du domaine religieux est caractérisée par le latin : "anima tua ", "liberar nos a malo ", " semper", " non est insipiens ", "bonum monasterium ", " dominum nostrum », " amen ». Nous avons écarté volontairement le syntagme nominal «Domini Nostri Iesu Christi » car les majuscules dans le texte l'isolent du reste des mentions latines. En effet, "dominum nostrum», en dépit de ce que Salvatore parle de Dieu, ne comporte pas de majuscules quand tout le syntagme précédent en porte à chaque mot. Il semblerait que ce soit un texte, plutôt qu'une parole, que le narrateur donne à lire au lecteur car, à l'oral, nous nous accorderons à dire qu'aucune différence ne peut apparaitre entre majuscules et minuscules au milieu d'une phrase et que seules les lois de l'écrit obligent le scripteur à en mettre derrière les signes d'assises doubles et le point final ${ }^{31}$. Le système linguistique de Salvatore peut donc assumer des lois telles que la dissimilation, qu'il marque en utilisant un vocable bien précis en fonction du référent qu'il veut représenter et évitant toute ambiguiité. Notons, par ailleurs, que les flexions latines sont employées

29 Traduction : mais.

30 Traduction : nommer, désigner. Dans le cas présent, le personnage de Salvatore étant investi d'une forte fonction thaumaturgique, les acceptions peuvent aller de pair dans les emplois $\mathrm{du}$ verbe "nominare " dans le texte original.

31 Ainsi, nous pourrions alors supposer que ce syntagme est à son tour une citation de quelque texte liturgique que Salvatore aura entendu lors des offices et que c'est ainsi qu'il nomme JésusChrist quand il dit seulement "dominum nostrum " pour se référer à Dieu. 
dans leurs justes fonctions, selon le cas : un génitif singulier après la particule "de" et un accusatif singulier après le verbe transitif "priega». Si ce langage prend en effet quelques libertés quant au système, il reste cependant tributaire des lois qui régissent chacun des systèmes mis en contact.

Une autre sphère est celle du temporel, qui s'oppose au spirituel. Lorsqu'il parle de joie, de plaisir ou de douleur, sentiments proprement humains, c'est dans des langues plus vernaculaires telles que le provençal, l'italien ou l'espagnol qu'il s'exprime ${ }^{32}$. La phrase la plus représentative de cette sphère est «Et anco jois m'es dols et plazer m'es dolors... ${ }^{33}$ " car sa relative homogénéitée ${ }^{34}$ idiomatique se détache des bribes disséminées dans les autres phrases, de même qu' elle se démarque par l'aposiopèse qui la clôt quand toutes les autres propositions relèvent du mode assertif ou exclamatif. Si le français propose une métathèse pour rapprocher les deux lexies, la langue vernaculaire employée par le personnage du roman se fait plus subtile : "dolors " apparaît comme portant une réduplication phonique de la voyelle [o] et de consonnes dont le point d'articulation est proche, le [r] apico-alvéolaire et le [1] palatal, toutes deux sonores et latérales. Cependant, «dols» (deuils) apparaît en hypogramme

32 Dans cette mosaïque de langues imbriquées au sein d'un même syntagme phrastique, le lecteur ne possède que des pistes lui permettant de distinguer les variétés linguistiques entre elles. Une connaissance des langues en présence facilite évidemment l'identification de celles-ci. Ainsi, un lecteur parlant l'espagnol et le latin pourra identifier le syntagme verbal "liberar nos " comme du latin, avec un pronom simplement postposé alors qu'en espagnol, on y trouverait une enclise, "liberarnos". Pour ce qui est du latin employé dans cet exemple, nous pouvons supposer qu'il s'agit d'un latin tardif puisque "liberare " est apocopé et devient "liberar " en préparation du phénomène d'enclise qui aura également lieu, par la suite, en italien.

33 Cet alexandrin, au-delà de l'aspect lyrique qu'il décrit, est un renvoi à la rhétorique courtoise. Il convient de rappeler que Dante et Pétrarque étaient contemporains de l'époque à laquelle se déroulent les faits décrits par Adso. Richard Dragonetti rappelle que Dante a proposé une certaine codification de cette rhétorique et met en exergue l'ornamento ou ornementation, qui correspond à l'elocutio cicéronienne, qu'il distribue en trois modes : grave, tempéré et familier que l'on adapte en fonction de l'objet du poème, de l'auditoire auquel on s'adresse et des circonstances dans lesquelles le trouvère compose. Dragonetti fait la remarque suivante : « La qualité de l'élocution dépend aussi de la condition sociale des personnages et la vraisemblance du style résulte alors d'une convenance entre les choses, les mots, les figures de style et la dignité des personnes dont on parle. ", in La Technique poétique des trouvères dans la chanson courtoise, Genève, Slatkine, 1979, p. 15-16. Pour codifier cette rhétorique, Dante s'est en partie appuyé sur les chansons des trouvères provençaux, langue à laquelle la majorité des mots de ce vers appartient. Ici, Salvatore devient le prototype de la rhétorique courtoise, son langage se faisant miroir de tout le spectre social dont il est issu. Notons que cette classification rhétorique place le comique au plus bas de la hiérarchie des genres, le rattachant au "vulgaire ", ce qui n'est pas sans rappeler les propos de Jorge de Burgos au sujet du rire. L'allusion à Pétrarque passe également par la lingua volgare dont le poète s’est fait la voix, rappelle des thèmes poétiques tels que la solitude, l'amertume et la vanité, largement représentés dans le Canzoniere.

34 Nous entendons par "relative homogénéité idiomatique " le fait que ce vers est majoritairement composé de provençal mais le mot «anco ", par exemple, est une forme archaïque du mot italien anche, aussi. 
complet dans "dolors" (douleurs), qui deviendrait alors une sorte d'amplification du premier $\operatorname{mot}^{35}$. La question de la motivation, déjà amorcée par le choix d'un vocable plutôt que d'un autre pour représenter une réalité donnée dans un contexte précis, se fait à nouveau jour à l'échelle du signifiant.

Le rapport de ces éléments entre eux est reflet du rapport que le sujet entretient avec le langage qu'il manipule : Salvatore nomme en vertu d'un système qui lui est propre et établit donc les rapports qu'il impose, à défaut de voir ceux qui s'imposent. Néanmoins, rappelons à cet endroit que si cette remarque est valable pour le discours de Salvatore, elle est également valable pour le discours d'Adso dont la dynamique, nous l'avons vu, est assurée par l'alternance d'une parole propre et d'une parole extérieure intimement liée à la sienne.

Un autre aspect de la langue de Salvatore est à prendre en compte dans cette première prise de parole : il s'agit des " approximations " de son langage qui sont transcrites telles quelles par la main d'Adso. À deux reprises, le moine se trompe et prononce des mots tels que "ve piase " au lieu de "vi piace" et "carcagna" en lieu et place de "calcagna " ${ }^{36}$. Une fois encore, tel l'enfant apprenant à parler qui ne peut que répéter ce qu'il entend, Salvatore demeure dans une écholalie et ne peut que restituer, parfois au prix d'approximations, que les sons qu'il entend sans autre support que celui de sa mémoire. Nous sommes alors en droit de nous demander si ces erreurs, lesquelles lui sont imputables à première vue, ne viennent pas en fait des locuteurs qu'il a croisés : il aurait alors entendu un mot erroné qu'il aurait mémorisé comme tel, son esprit tel une page blanche où la main d'autrui aura laissé une empreinte fausse que la main du scripteur n'aura pas rectifiée $e^{37}$.

35 Le rapprochement entre les lexies «dols» et " dolors» se fait également de façon étymologique. Le radical dol-, qui donne dolere (souffrir) en italien, doler en espagnol, mais aussi le mot français « deuil ». Cette remarque sur cette parenté apparente des langues employées et mêlées dans cette phrase témoigne d'une compatibilité résultant probablement du fait que toutes sont flexionnelles, soit participant d'une même famille linguistique. Corrélativement, ces langues semblent postuler l'idée d'une "hyperlangue romane " qui les regrouperait en amont de leur variations. Ainsi, le lecteur pourra faire le rapprochement entre le "plazer" (plaisir) provençal et le mot "placer" en espagnol, ou encore entre le mot " jois" et l'italien " gioia".

36 Il convient ici d'observer, d'un point de vue purement linguistique, les erreurs commises par Salvatore puisqu'elles semblent témoigner d'une motivation sur le plan actantiel. Le premier lapsus, dans "cargagna [sic.]", porte sur la sonorisation du [č] qui perd l'attaque occlusive et passe d'affriquée à fricative et le second porte, pour "vi piase [sic.]" sur la même paire de consonnes latérales que celles qu'il met en exergue dans la phrase qu'il prononce en provençal, à savoir les latérales sonores [l] et [r]. Ces erreurs étant mineures et ne nuisant pas à la reconnaissance des mots malgré les vices qui s'y cachent, nous pouvons considérer que ces «fautes de langue »- qui ne touchent que la langue italienne, soit la langue du contexte - participent à montrer la fragilité, voire la faillibilité d'un système linguistique hors normes tout en mettant l'accent sur la nature profondément humaine de cet " être » si difficile à cerner.

37 Une remarque est à faire ici : le latin, langue véhiculaire des hommes de savoir, n'est jamais malmené et les verbes tout comme les cas sont employés dans leur juste valeur. 
Cette opiniâtreté à transcrire le plus fidèlement possible les propos de Salvatore est assumée par le narrateur qui, dans une de ses nombreuses prolepse, l'annonce et s'y tient. "Dovrò, nel prosieguo di questa stroria, parlare ancora, e molto, di questa creatura e referirne $i$ discorsi. ${ }^{38}$ ", nous dit le moine autrichien, plaidant par ailleurs en faveur de l'importance réelle que le lecteur doit accorder à ces " discours " tant pour leur nombre que pour leur teneur. Malgré l'application que met Adso à reporter les moindres paroles de Salvatore, il évoque pourtant dans la glose de cette première manifestation langagière de la part de ce dernier une difficulté éprouvée à comprendre ce qu'il dit tout comme il lui est difficile de savoir quelle langue il parle. Devant l'aspect chaotique de ce "langage ", Adso a recours à un parallèle éloquent : "Era come se la sua favella fosse quale la sua faccia, messa insieme con pezzi di facce altrui ${ }^{39}$ [...] ». La description physique de Salvatore qui précédait son discours trouve ici toute sa motivation : elle s'inscrit dans le continuum métaphorique de son langage, versant incarné et tangible de cette langue faite de toutes et d'aucune.

Ce n'est pourtant pas avec Adso que s'amorce la première réelle interlocution de Salvatore. C'est à Guillaume, surgissant après ce monologue qui tient vraisemblablement du solipsisme, que revient la primauté de confronter sa langue structurée à celle, dispersée, de Salvatore. Il s' agit là de la seule occurrence de répétition d'un mot employé par l'assistant du cellérier. Une fois encore, ce n'est pas tant pour expliciter ce que Salvatore a dit et que Guillaume a parfaitement compris, mais bien pour attirer l'attention du lecteur sur quelque détail utile au bon déroulement des péripéties : "Perché hai detto penitenziagite. ${ }^{240}$ ". Si l'ancien inquisiteur demande à son confrère la raison pour laquelle il a employé ce mot, il apparaît qu'il incombe au lecteur de se préoccuper à présent de deux points : le passé et l'expérience de Guillaume, que le narrateur n’a pas encore dévoilés jusque là, et le passé et l'expérience de Salvatore, dont Adso ignore tout au moment où les deux hommes se rencontrent. Le mot "penitenziagite » est un pivot : pour Guillaume, il est le signe d'un passé hérétique probable de Salvatore tandis que pour Salvatore ${ }^{41}$, il est un signe d'appartenance dissi-

38 Eco Umberto, ibid., p. 46. Traduction : Il me faudra, dans la suite de cette histoire, parler encore, et beaucoup, de cette créature et en rapporter les discours.

39 Eco Umberto, ibid., p. 47. Traduction : C'était comme si son discours était pareil à son visage, fabriqué à partir de morceaux de visages d'autrui. Cette comparaison vient étayer l'hypothèse du narrateur quant au fonctionnement du système linguistique propre au personnage de Salvatore : il "fabrique " un discours à l'aide de morceaux de discours d'autrui.

40 Eco Umberto, ibid., p. 48. Traduction : Pourquoi as-tu dit penitenziagite?.

41 À l'instar du vers en provençal que nous avons rencontré plus haut, le mot "penitenziagite" et une formule en latin vulgaire, contraction de l'expression "poenitentiam agite ", soit "faites pénitence ». Il s'agit du cri de reconnaissance des Dolciniens (disciples du prêcheur Fra Dolcino, condamné au bûcher en 1307), secte condamnée comme hérétique par l'Église au XIVe siècle. Détournant les théories franciscaines sur la pauvreté du Christ, Fra Dolcino exhorta ses disciples à pratiquer la même vie apostolique que le Messie, condamnant publi- 
mulé dès que Guillaume le remarque. Le déni qui s'ensuit semble montrer que Salvatore a bel et bien saisi, par déduction, que Guillaume aura relevé ce mot car il en connaît le contexte d'usage. Cette négation passe par le malentendu, au sens littéral du terme, car Salvatore réactive aussitôt dans sa réponse une lexie déjà présente dans le texte: "Jesus venturus est et li homini debent facere penitentia. No ${ }^{2+2} "$. Outre la quête systématique d'assentiment dans les premières phases du dialogue avec le "No? " final récurrent, nous noterons la référence à Jésus-Christ et le passage au latin à la fin de la phrase qui se termine sur une paronomase qui n'est en fait qu'un glissement idiomatique. Salvatore veut perdre Guillaume dans le dédale de son langage, figurant le moment où Guillaume se perdra dans le labyrinthe de la bibliothèque.

C'est à partir de cette syllepse provoquant un malaise de part et d'autre que la situation interlocutive devient irréelle, voire fantaisiste. En effet, même si le lecteur, à l'instar du narrateur, ne sait rien du passé de l'un ou de l'autre des protagonistes du dialogue, il saisira néanmoins le renversement des rôles qui s'opère dans le discours des deux instances dialogiques. C'est Salvatore qui, lorsque Guillaume lui demande s'il provient d'un couvent de minorites, lui répond " no intendo $0^{43}$ ", brouillant ainsi le canal du code par son langage, tente aussi de rompre la fonction phatique. Paradoxalement, c'est le locuteur qui s'expose le plus à l'incompréhension qui la feint. Devant l'insistance de l'ancien inquisiteur, Salvatore murmure un "vade retro ${ }^{44}$ " qui s'applique à l'orthodoxie de Guillaume quand lui-même doit être suspecté d'hérésie, cette locution étant bien connotée dans un contexte religieux.

Or dans cette même situation de dialogue, le réseau des trames narratives se densifie : Adso, exclu de l'interaction, devient alors spectateur de la scène, tout comme le lecteur l'est au moment où il lit. C'est donc qu'Adso rapporte que Guillaume répète ce que Salvatore dit.

Revenons un moment sur les différentes sphères idiomatiques formant le système langagier de Salvatore. Si nous partons du postulat que la langue elle-même est vectrice de signifiance, elle s'intègre cependant dans un méta-

quement l'Église Romaine pour les richesses qu'elle avait accumulées et qui, selon sa doctrine, entraient en contradiction avec ce que les Saintes Écritures recommandaient. Ainsi, les Dolciniens se rendirent coupables de pillages et de saccages sanglants des biens appartenant au clergé. Ce mouvement hétérodoxe fit l'objet d'une répression sévère par l'Inquisition, notamment après l'expurgation du catharisme un siècle plus tôt.

42 Eco Umberto, ibid., p. 48. Traduction : Jésus viendra (littéralement, est à venir) et les hommes doivent faire pénitence. Non?

43 Eco Umberto, ibid., p. 48. Traduction : je ne comprends pas.

44 Eco Umberto, ibid., p. 48. Traduction : va-t'en. Cette formule consacrée, connue notamment pour être employée dans le Rituel Romain lors d'exorcismes ("Vade retro, Satana ", soit "Arrière, Satan!»), suppose ici l'idée sous-jacente d'un jugement de valeur incluant le Mal, mais détourné puisque c'est l'hérétique qui la prononce à l'adresse d'un ministre de Dieu et non l'inverse. 
système complet : un système capable de représenter et de d'adapter à toutes les réalités et même à l'insulte. En effet, le second jour à l'heure de Tierce, l'assistant-cellérier, devant la vision du cuisinier rabrouant les mendiants qui quêtent l'aumône, fait par à celui-ci de son mécontentement. Demandant à son confrère de faire preuve de miséricorde et de charité, deux vertus cardinales de la religion catholique, c'est en latin qu'il entreprend d'exposer son grief avant de revenir partiellement à la langue italienne : "Filii Dei, sono,", disse Salvatore, "Gesù ha detto che facite per lui quello che facite a uno di questi pueri!" 45 ". La spirale discursive est pourtant de nouveau amorcée : Adso rapporte que Salvatore paraphrase ce que Jésus-Christ a dit, notamment avec l'emploi du verbum dicendi au passé composé ( ha detto») qui signifie à la fois au cuisinier et au lecteur que les commandements mentionnés sont toujours en vigueur. Lorsque le cuisinier piqué au vif l'insulte en retour, Salvatore répond sur le même registre, reprenant les topiques avancés par le cuisinier. Ce dernier le traite d'hérétique : "Fraticello delle mie brache, scoreggione di un minorita! ${ }^{146}$ ". La réponse condense et réfute, de même qu'elle retourne contre lui les insultes du cuisinier : "Non sono un fraticello minorita! Sono un monaco Sancti Benedicti! Merdre a toy, bogomilo di merda! ${ }^{1 / 7}$ ". Rassemblant sous un même syntagme nominal ("fraticello minorita») les deux accusations du cuisiner portant sur son hétérodoxie religieuse, il rebondit également sur l'aspect scatologique avancé par le cuisinier ("scorregione ", dont le suffixe augmentatif a une valeur ici péjorative) en répétant deux fois le même mot de deux façons différentes : "merdre » qu'il doit sans doute tenir du français ${ }^{48}$

45 Eco Umberto, op. cit., p. 115. Traduction : "Les fils de Dieu, ils sont, ", dit Salvatore, "Jésus a dit que vous faites pour lui ce que vous faites à l'un de ces enfants!". La mention de Dieu est en latin mais la mention du passage sur le "Jugement denier» (Matthieu, 25:40), par son caractère paraphrastique, se fait selon le système idiomatique de Salvatore. En effet, il ne cite pas, il rapporte, rappelle ce qui a déjà été dit et qu'il n'a qu'entendu dire.

46 Eco Umberto, op. cit., p. 115. Traduction : Fraticelle de mes braies, pet de minorite. Les fraticelles (diminutif du mot italien frate, frère) désignaient des moines de l'ordre franciscain croyant en la pauvreté absolue du Christ et prescrivant une stricte observance de celle-ci, parfois jusqu’à entrer en conflit avec l'Église. Quant aux minorites, ils désignent plus largement les moines de l'ordre franciscain, également appelé "ordre mineur".

47 Eco Umberto, op. cit., p. 115. Traduction: Je ne suis pas un fraticelle minorite! Je suis un moine de Saint Benoît! Merdre à toi, bogomile de merde! En invoquant ici Saint Benoît, Salvatore oppose la branche franciscaine, creuset des hétérodoxies qui ont manqué de la conduire à sa simple destruction - et c'est sur ceci que porte la fameuse controverse qui a lieu dans le roman -, à la branche bénédictine, la plus ancienne, qui a engendré les ordres prêcheurs comme les Dominicains dont est issue l'Inquisition. Revendiquant son appartenance à l'ordre de Saint Benoît, puisqu'il vit maintenant dans une abbaye bénédictine, Salvatore pense ainsi écarter tout soupçon d'hérésie de sa personne et prouver de fait son orthodoxie.

48 Il semblerait que ce " merdre " prononcé par Salvatore soit effectivement une déformation du mot français et trouve sa place dans le langage babélique du moine bossu. Cependant, nous ne pourrons pas nous empêcher de penser à ce même juron dans la bouche d'Ubu, dans l'œuvre d'Alfred Jarry publiée en 1896, anachronisme plein d'humour qui convoque un texte autre dans le discours du personnage. 
et " merda " qu'il tient de l'italien, langue dans laquelle il a majoritairement évolué lors de la dispute, sauf pour revendiquer son statut de bénédictin. Apte à comprendre l'insulte, Salvatore a mis en place dans son système langagier, une série de membres de phrases qu'il peut remobiliser dans le même type de situation, comme un réflexe linguistique pertinent, et démontre ainsi la finitude de son œuvre thaumaturgique.

Il nous faut néanmoins tenir compte du fait qu'au-delà de sa fonction créatrice dans le langage, Salvatore assume également une fonction créatrice dans le récit. Il raconte des histoires qu'Adso transcrit à son tour en les identifiant comme telles : "[...] mi rancontò la storia della sua fuga dal villagio natio $^{49}[\ldots]$ ", "Cominciò a racontare una strana storia ${ }^{50}[\ldots]$ ". Ce que fait Salvatore est ce que l'Homme a fait à partir du moment où il a été en possession d'un langage articulé : inventer ou raconter des histoires. Témoignages des faits auxquels il a assisté, des situations qu'il a vécues, inventions, les histoires sont la manifestation d'une activité poétique du langage, laquelle peut alors ouvrir sur une dimension symbolique. Là encore, il pourrait effectivement s'agir d'une manœuvre d'emboîtement puisque l'auteur reporte qu'Adso nous raconte l'histoire que Salvatore lui a racontée. Le lecteur est double-allocutaire : celui de l'auteur et celui du narrateur.

Une autre caractéristique inhérente à la langue est la capacité d'évolution, elle aussi représentée dans les interventions dialoguées de Salvatore. Au début, il ne parle qu'un panel, somme toute réduit, de langues dites " romanes " et qui entretiennent entre elles quelques liens lexicaux ou syntaxiques. Nous y avons reconnu du latin, laquelle a donné les autres langues dont se sert l'assistant-cellérier : l'espagnol, l'italien, le français, le provençal. Si nous considérons à présent le deuxième passage dialogué entre Salvatore, il nous faut tout d'abord considérer son cotexte proche et plus particulièrement un échange qui a lieu entre Guillaume et Adso où le vieil homme, ayant manqué l'heure du repas, demande à son novice d'aller chercher quelque nourriture aux cuisines, se heurtant à la stupeur d'Adso, qui croit avoir compris que son maittre lui demandait de voler leurs pitances. C'est ainsi que Guillaume, en justifiant sa demande, explicite une mutation dans la relation entre Adso et Salvatore : "Chiedere. A Salvatore, che è ormai tu amico"s. ". Le verbe "Chiedere " à l'infinitif fait écho à "Rubare. \$2 " prononcé juste avant par le jeune homme : à l'action "criminelle " se substitue donc la parole. Néanmoins, en attribuant à Adso le statut d'ami de Salvatore, l'ancien inquisiteur pose les jalons du dialogue à suivre. Alors que Salvatore propose au novice une recette de son

49 Eco Umberto, ibid., p. 177. Traduction : [...] il me raconta l'histoire de la fuite de son village natal [...].

50 Eco Umberto, ibid., p. 253. Traduction : Il commença à me raconter une étrange histoire[...].

51 Eco Umberto, ibid., p. 206. Traduction : Demander. À Salvatore, qui est maintenant ton ami.

52 Eco Umberto, ibid., p. 206. Traduction : Voler? 
cru, le lecteur n'aura pas manqué de remarquer l'incursion de " $d u b i s^{53}$ " dans la recette et, tandis qu'il donne à Adso une lampe que ce dernier n'a pas demandée, l'assistant-cellérier justifie son geste : "Fileisch tuo magister vuole ire in loco buio esta noche ${ }^{54}$.". Aux constantes des langues romanes, s'ajoute un nouveau paradigme linguistique, celui de l'allemand. Or, même si de nombreuses pages ont été lues depuis le début du récit, le lecteur aura sans doute gardé à l'esprit qu'Adso, venant de Melk, est autrichien et que sa langue est donc l'allemand. Ainsi, tandis que les deux hommes nouent des liens de confiance, ceux-ci semblent se matérialiser dans la glaise systémique du langage de Salvatore ${ }^{55}$. Une autre hypothèse est envisageable cependant : un autre Allemand fait également partie de la confrérie et il s'agit du bibliothécaire, Malachie de Hidelsheim. En donnant la lampe à Adso sans que ce dernier l'eût demandée, Salvatore montre la voie de la résolution des crimes en indiquant un lieu sombre or il faudra passer par les catacombes pour accéder à l'Édifice et entrer dans la bibliothèque. Aussi, l'adverbe "fileisch » ouvrant sur une supposition de Salvatore dans la langue d'Adso encourage le lecteur à penser que l'hypothèse pourrait être celle d'Adso lui-même car elle apparaît formulée dans sa propre langue.

Enfin, considérons un dernier point ayant trait au langage de Salvatore. Au moment où le jeune novice est envoyé par son maître pour ramener leurs repas, Adso trouve Salvatore dans l'écurie. S’extasiant devant la beauté du cheval de l'abbé, le jeune bénédictin confie à son " ami » qu'il aimerait bien le monter. Salvatore le détrompe aussitôt et détourne une fois encore le propos : "Anco quello sufficit... Vide illuc, tertius equi... ${ }^{56}$ ", que suit immédiatement l'explication d'Adso : "Voleva indicarmi il terzo cavallo. Risi del suo buffisimo latino $^{57}$ ». Notons tout d'abord que, lors de ce dernier véritable échange entre

53 Traduction : tu es. Il s'agit d'une transcription phonétique de «du bist » que le transcripteur n’a pas corrigée.

54 Eco Umberto, ibid., p. 207. Traduction : Peut-être que ton maître veut aller en quelque lieu sombre cette nuit. L'incursion de l'allemand ne va pas sans anicroche puisque le mot "fileisch" est ici retranscrit de façon purement phonétique. Adso, pourtant germanophone, n'a pas corrigé l'erreur lors de sa transcription et n'a pas remplacé le mot erroné par "vielleicht ", signifiant peut-être, dans le but de demeurer fidèle aux paroles de Salvatore tel qu’annoncé au début du roman.

55 C'est pourquoi le lecteur est alors en droit de supposer qu'il y aura sans doute eu d'autres échanges, non rapportés, entre les deux personnages au cours desquels Salvatore aura peut-être entendu le jeune novice s'exprimer dans sa langue maternelle.

56 Eco Umberto, ibid., p. 206. Traduction : Même celui-là [le cheval] suffit... Regarde celui-là, le troisième cheval...

57 Eco Umberto, ibid., p. 206. Traduction : Il voulait mindiquer le troisième cheval. Je ris de son latin drôlissime. Ce qui fait rire Adso, c'est la confusion grammaticale que fait Salvatore. S'il voulait désigner le troisième cheval, il aurait dû dire "tertium equum ». En disant "tertius equi ", il fait un non-sens que l'on pourrait traduire par le " troisième de cheval " puisqu'il avait voulu désigner le tiers d'un cheval, il aurait dû dire "tertium equii ». 
les deux protagonistes du dialogue, c'est Adso qui rit en dépit de l'interdiction formelle de rire que comporte la Règle de son ordre et que le vieux Jorge de Burgos a rappelé en latin lors de la discussion que eut lieu dans le scriptorium avec Guillaume au début du récit. Si Salvatore a appris l'allemand d'Adso, Adso a appris de Salvatore à enfreindre les règles, créant ici encore une sorte de réciprocité sur des plans différents. Ensuite, si le novice semble se moquer du latin approximatif de l'assistant-cellérier, c'est en effet parce qu'il y a de nouveau un malentendu au sens propre du terme. Il ne s'agit pas d'une syllepse mais bien d'un louchement, qui se mue en une forme de prolepse. Sur l'instant, Adso a bien compris que Salvatore désigne le troisième cheval. Lorsque Salvatore meurt et qu'Adso évoque le souvenir de cet épisode en repassant devant l'écurie, il se remémore l'anecdote et sourit : le sourire se pose comme une forme de signifiant non-verbal dont le référent serait alors Salvatore. Ce sourire ayant surpris Guillaume, ce dernier demande à son novice la raison de ce manquement à la discipline et Adso change alors son interprétation : "Niente, mi ricordavo del povero Salvatore. Voleva fare chissà quale magia con quel cavallo, e col suo latino designava come tertius equi. Che sarebbe la $\mathrm{u}^{58}$. ". A la façon d'une charade, Adso offre une nouvelle représentation du "tertius equi " de Salvatore, pris au pied de la lettre : le troisième/tiers "du cheval ", soit la troisième lettre de "equi " en latin, qui est le $u$. C'est de cette "sottise" d'Adso que Guillaume déduira la clé de l'énigme qui les empêche de toucher au Finis Africae de la bibliothèque : en rectifiant la signification de la note de Vénantius comme Adso a rectifié la signification du mot "tertius », il pourra actionner le mécanisme qui le conduira à l'endroit de l'Édifice où sont entreposés les livres mis à l'index. En outre, c'est une mise en évidence du caractère méta-linguistique de toute langue que postule la manipulation à laquelle se livre Guillaume, consécutivement à Adso : "[...] primum et septimum de quatuor non significa il primo e il settimo dei quattro, ma del quattro, della parola quattro ${ }^{59}$ ! ". Le mot " parola " apparaissant de façon explicite, le doute n'est plus permis quant à la portée auto-réflexive du langage tel qu'envisagé dans le roman d'Umberto Eco. Cet avatar linguistique étant délicat à restituer à l'écran, c'est sur l'anglais "four» que Guillaume fonde son raisonnement et qu'il différencie du quatuor latin non pas à cause du sens mais bien du nombre de lettres. En l'absence d'une analogie aussi prégnante entre le latin et l'italien qui comptent le même nombre de lettres dans quattro et quatuor, c'est leur nombre qui devient vecteur de signifiance pour Guillaume, non pas dans l'analogie mais bien dans la différence.

58 Eco Umberto, ibid., p. 432. Traduction : Rien. Je me rappelais du pauvre Salvatore. Il voulait faire je-ne-sais quelle magie avec ce cheval qu'avec son latin il désignait comme le troisième de cheval. Qui serait le u [dans equi]. Ici, Adso ne fait que "prendre au mot " ce qu'a dit Salvatore et pousser plus avant son inconséquence syntaxique.

59 Eco Umberto, ibid., p. 432. Traduction : [...] la première et la septième des quatre ne signifie pas la première et la septième des quatre, mais du quatre, du mot quatre! 
De même, le support multi-canal qu'est l'adaptation cinématographique du roman n'offre que peu de prise à la mise en relief de la dimension métatextuelle omniprésente dans le roman du linguiste italien. Aussi, le film s'ouvre sur un inter-titre : "A palimpsest of Umberto Eco's novel " (0:00:59), plaçant à la fois le film sous l'augure de la citation, de la restitution mais aussi d'une intertexualité interne à l'auteur puisque le spectateur ne manquera pas de faire le lien entre ce message à l'image et l'intérêt du romancier pour les palimpsestes, notamment dans Lector in fabula paru en 1979. Dans le film, le Beatus de Liebana est mentionné par Guillaume quand il trouve enfin la bibliothèque, ce qui n'est pas sans rappeler l'ouvrage qu'Umberto Eco consacra à ce manuscrit du Moyen-Âge et qui parut en 1982.

D’autres signes de cette poétique de l'auto-réflexivité apparaissent cependant dans l'adaptation cinématographique du livre. Lorsque Guillaume, et non Adso, finit par questionner Salvatore directement au sujet des morts dans l'abbaye, il le fait dans le cimetière où Salvatore chasse des rats. Toujours selon les canons de l'esthétique médiévale, Guillaume se trouve dans le même champ que Salvatore, tout deux en pieds, mais l'ancien inquisiteur se trouvant sur la partie de la butte qui domine celle où se trouve Salvatore, il apparaît plus grand que ce dernier aux yeux du spectateur. Quant aux rats que l'ancien hérétique chasse, Guillaume se voit confirmer que son confrère les capture dans l'intention de les manger (0:47:03). Or les deux apparitions précédentes des rats ont eu lieu dans le cadre de la bibliothèque : ce sont les rongeurs qui permettent au vieil homme ainsi qu’à son novice de localiser les livres dans l'Édifice. De fait, les rats mangeant les livres, Salvatore mangeant les rats mange effectivement des livres. D’une certaine façon, à défaut de pouvoir s'approprier le savoir par la lecture, il l'ingère comme nourriture de son enveloppe charnelle, laquelle " nourrit ». Quelques secondes plus tard, Guillaume " usurpe " à nouveau le statut d'Adso quand il rapporte au style indirect ce qui a été éludé à l'écran, reformulant ce qui n’a finalement pas été entendu (0:47:46). Enfin, lorsque Salvatore lui répond, il bute sur le mot "translator" qu'il n'arrive pas à prononcer ou dont il ne se souvient plus, proposant à la place une lexie plus «étymologique " avec le mot de "translogo» (0:47:52). Ici encore, le rappel semble manifeste : Salvatore échoue précisément à nommer la figure qui incarne la rigueur systémique et l'hermétisme des langues. Pour le traducteur, les langues ne s'imbriquent pas : elles coexistent et son savoir lui permet de passer de l'une à l'autre, de l'une dans l'autre.

\section{Réécrire le(s) mythe(s)}

En revanche, ce que l'adaptation cinématographique rend de façon sans doute plus visible que le roman, c'est la polarisation axiologique dont Salvatore fait l'objet. Dans le livre, le moine difforme perd peu à peu son 
apparence d'" animal » au profit de l'homme qu'il est. Mais c'est au prix d'une lente reptation que le lecteur doit lire entre les lignes. Or à l'écran, alors que toute la tension narrative se focalise sur l'intrigue policière, Salvatore continue pourtant à jouer un rôle capital dans la résolution de l'enquête. Animal diabolique quand il apparaît pour la première fois à l'écran, il finit brûlé tel un martyr dans le film, passant ainsi de l'infamie de l'hérésie au châtiment des Saints. La caméra fait alors un plan sur les trois bûchers, celui de la jeune fille accusée de sorcellerie, celui du cellérier accusé d'hérésie et enfin, celui de Salvatore dont le seul tort aura été de répéter ce qu'il avait entendu (1:56:05). C'est le bûcher de Salvatore que l'on allume en premier : il est alors perçu à l'écran comme un halo de lumière, au bout de la diagonale des trois brasiers, dont la ligne est orientée vers la bibliothèque, laquelle prendra feu peu après. Au plan d'avant (0:51:00), la vue en plongée montre la torche qui embrase le fagot, laquelle réactive le feu de la lampe donnée à Adso. Au-delà de la figure christique que nous évoquions au début de notre analyse, c'est le mythe de Prométhée que semble à présent incarner Salvatore, repris à son tour par la mythographie chrétienne avec Lucifer (dont le nom prend ici tout son sens).

Par ailleurs, Salvatore est un des seuls personnels de l'abbaye à pouvoir se déplacer de jour comme de nuit entre l'intérieur et l'extérieur de l'Édifice, ce qui contribue à lui conférer un statut à mi-chemin entre l'humain et le divin, faisant de lui un actant purement transformationnel ${ }^{60}$.

Ambivalent comme tout symbole, Salvatore se trouve à la croisée entre deux mythes linguistiques : celui de la langue adamique et celui de la langue babélique. Prenant de la première la motivation et de la seconde l'arbitraire, il est également la cristallisation de l'Unique face au Divers, reprenant à son compte un débat de longue date qui continue à faire rage entre les anthropologues du langage. Nénamoins, Adso tranche en faveur de l'aspect babélique :

\section{[...] e una volta pensai che la sua [lingua] fosse, non la lingua adamica che l'uma- nità felice avevo parlato, tutti uniti da una sola favella, dalle origini del mondo sino alla Torre de Babele, e nemmeno una delle lingue sorte doppo il funesto evento della loro divisione, ma propio la lingua babelica del primo giorno dopo il castigo divino, la lingua della confusione primeva ${ }^{6}$.}

60 Le terme est emprunté à Jacques Fontanille dans Sémiotique du discours, Limoges, PULIM, 2003, p. 158 : «La logique des forces est une logique transformationnelle: elle définit les actants uniquement à partir de leur participation à une transformation entre deux états, et de leur engagement en vue de cette transformation. ». Les deux états dont il est question dans le roman que nous analysons sont l'ignorance et le savoir.

61 Eco Umberto, op. cit., p. 47. Traduction : [...] et une fois je pensai que la sienne [sa langue] fût, non la langue adamique que l'humanité bienheureuse avait parlé, tous unis par un seul discours, des origines du monde mais plutôt celle de la Tour de Babel, et même pas une des langues générées après le funeste événement de leur division, mais bien la langue babélique du premier jour après le châtiment divin, la langue de la confusion première. 
Sous couvert de l'hypotypose qui contribue à renforcer la monstruosité de Salvatore, représentation de chair et d'os du châtiment divin, c'est l'hypothèse linguistique de l'Un qui transparaît. Le parallèle établi entre le visage de Salvatore et la langue babélique qu'il parle, faite de lambeaux d'autres langues, ne saurait manquer de convoquer un autre texte : le Frankenstein de Claire Bampthon et Mary Shelley, paru en 1818 et dont le sous-titre était "Le Prométhée moderne ». Même dans les allusions intertextuelles, force est de constater que nous retrouvons ce jeu de "ricochets " qui, concentriques, ramènent tous au/aux récit(s).

Notons également que ce dernier rit à l'écran lorsqu'il est enduit de poix (1:50:39) et qu'il chante lorsqu'on lui demande de renier le Diable : comme dans le roman, Salvatore détourne à l'écran la situation commuincationnelle idéale et, à l'instar de la connivence qu'il établit dans le roman entre Adso et le lecteur qui se trouvent dans la même situation d'incompréhension, il fédère Adso et le spectateur dans le film puisque tous deux ne sont que "simples témoins " de la scène. Néanmoins, ce rire de Salvatore, omniprésent dans le roman comme dans le film, sont un renvoi perpétuel au second tome de la Poétique d'Aristote, dont le véritable auteur n'est autre que le romancier lui-même dans le contexte exclusif du récit.

Pour conclure, nous devons poser qu'aux deux notions du binôme « texte/ contexte " sur lequel se fonde notre réflexion et l'analyse du roman d'Umberto Eco, nous devrons ajouter celles d'intertexte comme un " ensemble de textes liés par des relations intertextuelles ${ }^{62} "$. Par le biais de la citation, c'est tout un panorama littéraire et culturel qui se dessine sous la plume d'un moine ayant vécu il a de cela plusieurs siècles où la chronologie n'importe plus. Des œuvres contemporaines côtoient des œuvres classiques ou antiques sans plus poser de problème quand l'énonciation se distancie du récit grâce à une chronologie fausse que l'on accrédite comme vraie. À ce propos, nous rappellerons les propos de Gérard Genette sur la Poétique d'Aristote, qui trouvent ici toute leur place : "Une première opposition est celle qu'indique Aristote en quelques phrases rapides dans la Poétique. Pour Aristote, le récit (diégésis) est un des deux modes de l'imitation poétique (mimésis), l'autre étant la représentation directe des événements par les acteurs parlant et agissant devant le public ${ }^{63} »$. Le tour de force du roman du linguiste italien est d'arriver à faire coexister au sein d'un même texte les deux modes : le récit comme contexte discursif, la représentation par les actants comme contexte énonciatif. Le texte, à l'instar du lecteur, est donc mis en tension entre ces deux pôles, basculant tantôt vers l'un tantôt vers l'autre sans jamais rester définitivement dans l'un des deux « camps».

62 Maigueneau Dominique, Les termes clés de l'analyse du discours, Paris, Seuil, 2009, p. 79.

63 Genette Gérard, Figures II, Paris, Seuil, 1969, p. 50. 
Quant au langage employé par Salvatore, il tend à confirmer une hypothèse avancée par Claude Hagège au sujet de la traduction : "Les langues different non par ce qu'elles peuvent ou non exprimer, mais par ce qu'elles obligent ou non à dire $e^{64}$. ". Ce langage a été conçu, nous l'avons vu, dans l'urgence de la situation communicationnelle. Des bribes de toutes les langues sont contiguës et font sens au sein d'un même syntagme, au sein d'un même axe des successivités. Peut-être faut-il y voir l'ébauche d'un pidgin tels qu'en conçurent les premiers hommes dotés du pouvoir de parole, récupérant les noyaux opérants des langues véhiculaires avec lesquelles ils entraient en contact, mus par la nécessité des transactions utiles à leur survie. Taxé d'hérétique, c'est sans aucun doute pour échapper aux fers et au bûcher que Salvatore ruse, déploie tout un système qui lui est propre pour ne faire comprendre que ce qu'il veut, et non ce qu'il peut. À l'inverse, il se refuse à comprendre ce qui pourrait le compromettre et engager sa survie. Dans sa conception du monde, langage et essence sont intrinsèquement liés. C'est sur ce principe même que se fonde la notion de récit : n'existe dans le récit que ce qui est nommé, re-présenté en différé et de façon pérenne par le canal de l'écrit. Cette fonction de l'écriture apparaît également comme un manifeste poétique : le roman "sature " la mémoire quand la poésie demande à être retenue or les renvois dans la trame narrative ne peuvent être perçus que si le lecteur a la possibilité de revenir sur ses pas, de re-lire et de re-lier les événements entre eux grâce au support écrit. C'est également ce support écrit qui permet de « jouer » avec la casse, les graphies, les langues spectrales qui apparaissent fugacement avant d'être emportées par un tourbillon archi-systémique. Le texte est alors image du texte et c'est au lecteur qu'il incombe de tirer cette conclusion à partir des différents procédés de dénudation mis en œuvre tout au long du récit afin de motiver le récit lui même :

Pourquoi la dénudation du procédé existe-t-elle ? Le procédé perceptible ne se justifie esthétiquement que lorsqu'il est volontairement rendu perceptible. Un procédé perceptible, masqué par l'auteur, produit une impression comique (aux dépens de l'œuvre). Prévenant cette impression, l'auteur révèle le procédé65.

Ce serait donc pour en assurer la vraisemblance et contraindre le lecteur à adhérer au pacte de lecture que l'on " mettrait à nu " les arcanes du récit selon Boris Tomachevski. Nous nuancerons ce propos en revenant au constat de départ de notre hypothèse, à savoir le texte perçu comme " entité irréductible " au sein duquel les signes entretiennent des rapports générateurs de sens pour ajouter à la juste proposition de Boris Tomachevski que le texte, en une sorte de réflexe préétabli, se révèle toujours dès lors qu’il est lu car il repose sur cette

64 Hagège Claude, L'Homme de parole, Paris, Arthème Fayard, 1985, p. 49.

65 Tomachevski Boris, "Thématique ", in Todorov Tsvetan, Théorie de la littérature, Paris, Seuil, 1965 , p. 301. 
combinaison de signes et se sustente d'elle. Ce qui est « livré en pâture » à l'interprétation du lecteur ne saurait être autre chose qu'une des nombreuses mailles du " tapis ", pour reprendre l'image de Nadine Ly, ou bien encore une case de l'échiquier si cher à Ferdinand de Saussure. Ainsi, à la question " Si le texte parle de lui-même, de quoi parle le récit ? ", nous répondrons simplement que le récit étant un texte, il parle avant tout de lui-même et parle à son lecteur ${ }^{66}$, la virtuosité de l'auteur ne s'exerçant que dans la manière dont il en parle.

Hynde Benachir

EA 3656 AMERIBER

Université Bordeaux Montaigne

hynde.benachir@etu.u-bordeaux-montaigne.fr

\begin{abstract}
Résumé
Cet article vise à mettre en lumière les enjeux du binôme texte/contexte appliqué aux interventions dialoguées du personnage de Salvatore dans le roman Il Nome della rosa, d'Umberto Eco. Marquées par l'idiosyncrasie ainsi qu'une forte dimension idiolectale, ses prises de parole sont à la fois la manifestation de la textualité comme inhérente au texte qu'elle transforme en objet d'analyse, du caractère motivé de tout acte de dénomination et, enfin, la matérialisation d'une polaristion axiologique qui élève Salvatore au rang de symbole. Pour compléter le champ de notre étude, nous tiendrons également compte de ces mêmes interventions dialoguées dans le cadre de l'adaptation filmique du roman afin d'illustrer au mieux la « texture " d'un récit aux allures de labyrinthe.
\end{abstract}

\title{
Mots-clés
}

Idiosyncrasie, dénomination, autoréférence, poétique du discours, sémiotique.

\begin{abstract}
This article intends to underline the stakes of the text/context binomial applied to the conversational interventions of the character Salvatore in the novel Il Nome della rosa, by Umberto Eco. Marked by idiosyncrasy as well as a strong idiolectal dimension, his speaking is at the same time the manifestation of textuality as inherent to the text it turns into an object of analysis, of the motivated nature of every act of denomination and, finally, the materialization of an axiological polarization that raises Salvatore to the level of a symbol. In order to complete the field of our study, we will also take into account the same conversational interventions in the context of the filmic adaptation of the novel in order to better illustrate the "texture" of a maze-like narrative.
\end{abstract}

\section{Keywords}

Idiosyncrasy, denomination, self-reference, poetics of speech, semiotics.

66 Umberto Eco rappelle le fait suivant : La différence référentielle (et par suite la valeur de la proposition) ne réside pas dans l'expression mais dans le récepteur. ", in L'Euvre ouverte, Paris, Seuil, 1965, p. 51. Plus loin dans son essai, le sémiologue écrit : «[...] le renvoi sémantique ne s'épuise pas dans la référence au denotatum, mais s'enrichit chaque fois que l'on jouit de la manière irremplaçable dont il fait corps avec le matériau qui lui donne sa structure ; la signification revient continuellement sur le signe et s'enrichit ainsi d'échos nouveaux. », op. cit., p. 55. 